\title{
Risk Assessment for Heavy Ions of Parts Tested with Protons
}

\author{
P.M. O'Neill, G D. Badhwar, and W.X. Culpepper, Member, IEEE \\ NASA Johnson Space Center, Houston, Texas 77058-3696
}

\begin{abstract}
An internuclear cascade - evaporation code is used to model energy deposition in thin slabs of silicon. This model shows that protons produce a significant number of events with effective Linear Energy Transfer (LET) greater than $8 \mathrm{MeV} \mathrm{cm} / \mathrm{mg}$ and demonstrates that proton testing of microelectronic components can be an effective way to screen devices for low earth orbit susceptibility to heavy ions.
\end{abstract}

\section{Introduction}

Testing the single event effect susceptibility of components using energetic (25-600 MeV) protons is often much less expensive than heavy ion testing. Parts are operated in air and do not need to be de-lidded when exposed to the protons, whereas heavy ion testing is usually performed in a vacuum on delidded parts. System level testing is practical with high energy protons since the beam is very penetrating. For example, at $200 \mathrm{MeV}$ only $10 \%$ energy loss occurs for a thickness of $5 \mathrm{~g} / \mathrm{cm}^{2}$ and that is almost $2 \mathrm{~cm}$ of aluminum. The $200 \mathrm{MeV}$ proton beam at the Indiana University Cyclotron Facility [1], [2] has a diameter up to 3 inches which makes system level testing very efficient. For programs such as Space Station and Shuttle, a large number of commercial - off - the - shelf (COTS) components are used in commercially available avionics that has not been designed for space environments. It is not practical (or even possible in most cases) to test each of these components individually.

The proton upset cross section is very useful for predicting the part upset rate for proton environments such as the South Atlantic Anomaly or solar flare protons [3]. However, proton upset cross section can not be used to accurately predict upsets due to heavy ions such as those found in Galactic Cosmic Rays (GCR) [4].

The following analysis shows that such a broad spectrum of equivalent heavy ion LET is produced by protons that a single LET threshold cannot be determined by proton testing. However, this broad spectrum can be used to identify failure modes that would occur by testing with heavy ions at LET's up to about $8 \mathrm{MeV} \mathrm{cm} / \mathrm{mg}$. This analysis is based on internuclear cascade and subsequent nuclear particle evaporation models whose accuracy has been demonstrated [5]-[8].

\section{Nuclear Interaction Model for Energetic Protons on Silicon}

The direct ionization Linear Energy Transfer (LET) for protons in silicon is much smaller than that for higher charged particles since LET increases as the square of the ion charge. However, occasionally the proton interacts with the silicon nuclei and produces a shower of fragments and a recoiling nucleus. When this happens, the energy deposited per unit length (effective LET) is much greater than the direct ionization LET.

Energy deposition in thin silicon slabs by fragments of the proton - silicon interaction has been studied previously [5][8]. These authors describe the interaction as a two stage process. In the cascade stage, the proton collides with the individual nuclei of the silicon, knocking out nuclei, imparting energy to the remaining recoil nucleus, and exciting the nucleus. This stage is modeled by a Monte Carlo simulation referred to as an internuclear cascade code. In this work, we have used the CLUST code [9], [10] to model the cascade stage. In the second stage, the excited nucleus evaporates particles in random directions until it is in the ground state. We have coded the algorithm provided by Tang [7] to model the evaporation stage (EVAP).

Using the CLUST-EVAP Code, we generated interaction sites at random within bulk silicon. We tracked each fragment particle emitted from the cascade process, the evaporation stage and finally the remaining recoil nuclei. Figure l shows

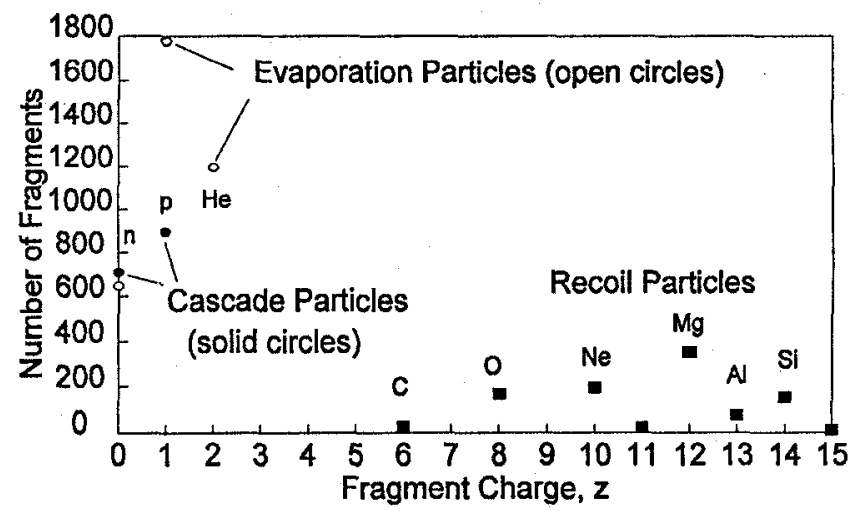

Figure 1. Number of the fragments produced by 1000 proton -silicon inelastic interactions for a proton energy of $200 \mathrm{MeV}$ versus fragment charge 
the charge distribution of fragments produced by 1000 proton - silicon inelastic interactions for a proton energy of 200 $\mathrm{MeV}$. For this proton energy, the cascade stage only produces neutrons and protons. The evaporation stage produces as many neutrons, about twice as many protons, and a large number of helium. Following these stages, the recoiling nuclei are composed of carbon to silicon, but mainly oxygen, neon, magnesium, and silicon. This shows that the average collision produces about 5 charged particles: 1 cascade and 2 evaporation protons, 1 evaporation helium, and 1 recoil nucleus. This suggests that a single ion, such as an evaporation helium or one of the recoils, as opposed to a shower of smaller particles, is responsible for most of the energy deposition in thin layers of silicon and thus single event effects.

The angular distribution of the fragments are shown in Figure 2. The cascade particles are concentrated in the forward direction and this is independent of incident proton energy.

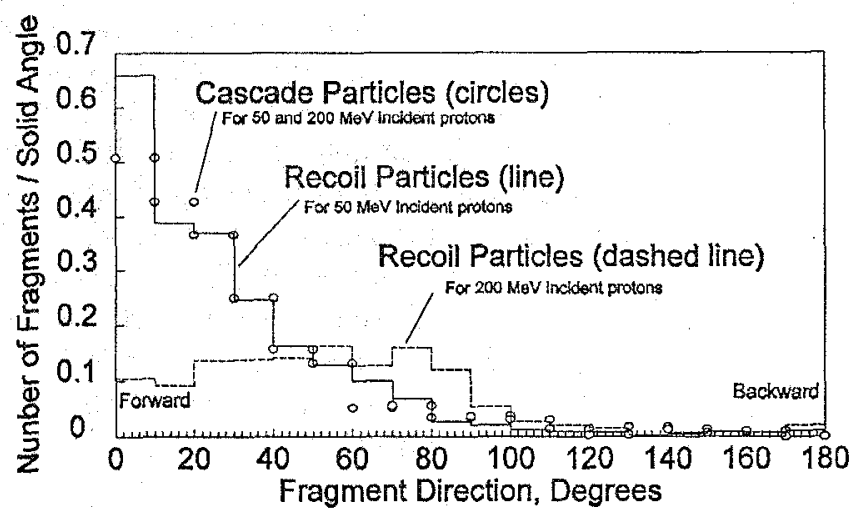

Figure 2. Angular distribution of fragments for 50 and $200 \mathrm{MeV}$ incident protons. The recoils are more isotropic for the $200 \mathrm{MeV}$ incident protons.

The angular distribution of evaporation particles is not shown because it is isotropic since particles are boiled off at random. The angular distribution for the recoiling nuclei for $50 \mathrm{MeV}$ incident protons is very close to that of the cascade particles (i.e. forward). This is because for lower incident proton energies about two-thirds of the cascade events result in a compound nucleus - the proton sticks to the nucleus and it is excited but there are no cascade particles. In this case the incident proton's momentum is all transferred to the nucleus. For $200 \mathrm{MeV}$ protons, compound nucleus formation is rare and the recoil is pushed backwards (180 degrees) by the generally forward (zero degrees) escaping cascades. Also, 200 $\mathrm{MeV}$ protons excite the nucleus more and produce more evaporation particles. This tends to flatten the angular distribution of the recoil particles as shown in Figure 2.

The distribution of the range in silicon of the fragments is shown in Figure 3. The range varies widely for cascade, evaporation, and recoil fragments. The cascade protons are the most penetrating followed by the evaporation fragments

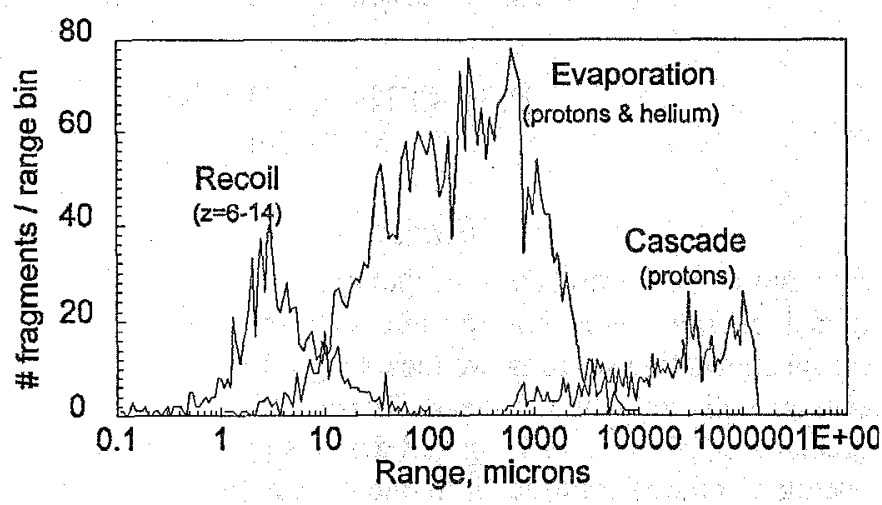

C:ISIGMAIFRAGRNG.G

Figure 3. Distribution of the range in silicon of cascade, evaporation and recoil fragments for $200 \mathrm{MeV}$ incident protons.

which consist of ions up to helium. The recoil nuclei generally have much lower range and they have higher charge (carbon to silicon). It is these recoils that are most responsible for energy deposition in thin layers of silicon.

\section{Effective LET Derived from Nuclear Interaction Model}

In the previous section we have shown that the CLUST EVAP nuclear interaction model predicts that relatively short range, individual ion (rather than a shower) fragments are responsible for energy deposition. Therefore, we have assumed a silicon slab located at the center of a cube of silicon as shown in Figure 4. We allow fragments from over 10 microns away (the typical recoil range) from the slab to deposit energy in it.

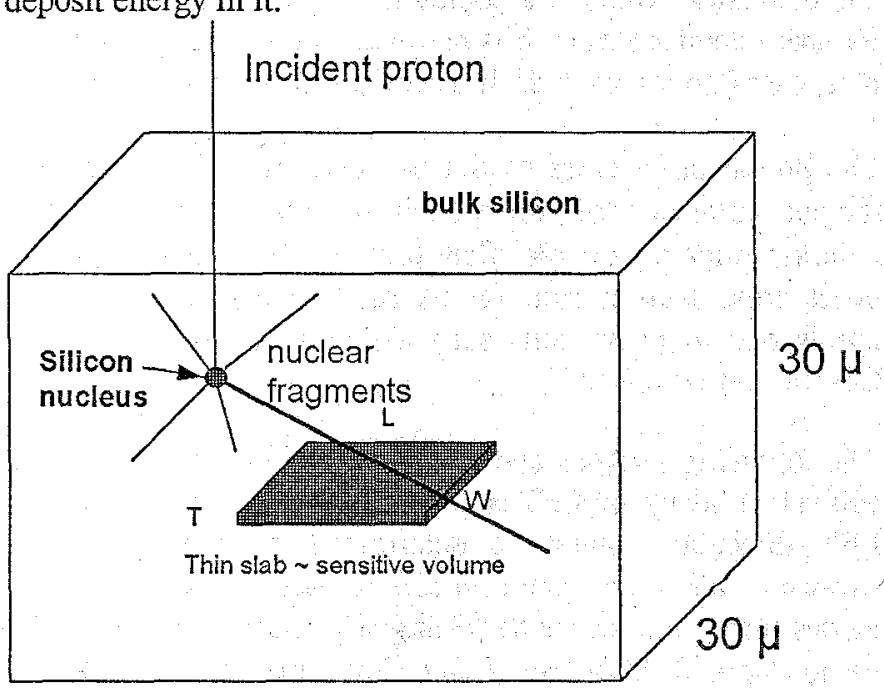

\section{$30 \mu$}

Figure 4. Fragments of the proton - silicon interaction deposit charge within a thin slab

Generating interaction sites at random within the bulk silicon, we calculated the energy deposited per unit thickness 
of the silicon slab (this defines effective LET for our purposes) for many (over $10^{6}$ ) events. Using this definition of effective LET, Figure 5 shows the differential LET spectrum for 50 and $200 \mathrm{MeV}$ incident protons for a 1 micron thick slab with sides of 10 microns. This geometry is typical of the sensitive volume for older technologies $(\sim 1$ micron). The differential LET spectra in Figure 5 are broad. The spectra's

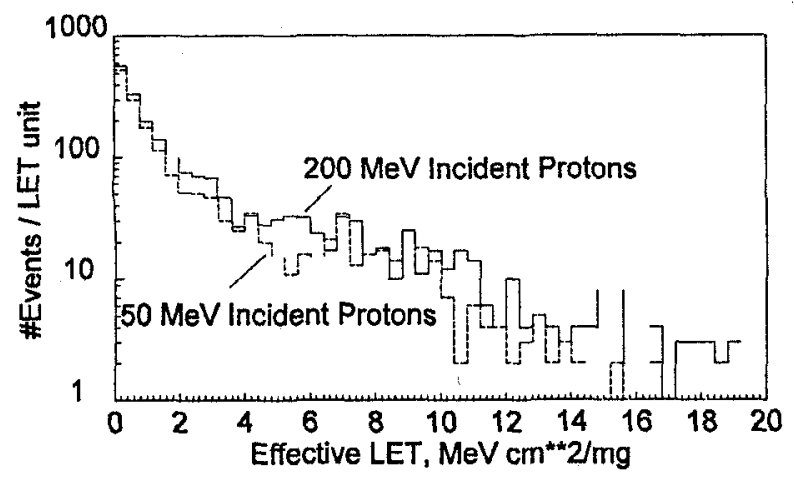

Figure 5. Differential LET spectra for thin slab ( $10 \times 10 \times 1$ micron) embedded in bulk silicon for 50 and $200 \mathrm{MeV}$ incident protons.

breadth is a manifestation of the large number of possible outcomes of the proton-silicon interaction and this feature limits the accuracy of associating effective LET with proton energy. Note that even though we generated the LET spectra for a thin slab thickness of 1 micron with 100 micron $^{2}$ crosssection, the spectra are very similar for much smaller geometries representative of newer technologies. Figure 6 shows the differential LET spectrum for $200 \mathrm{MeV}$ incident protons for a 1 micron thick slab with sides of 1 microns (geometry typical of the sensitive volume for newer technologies).

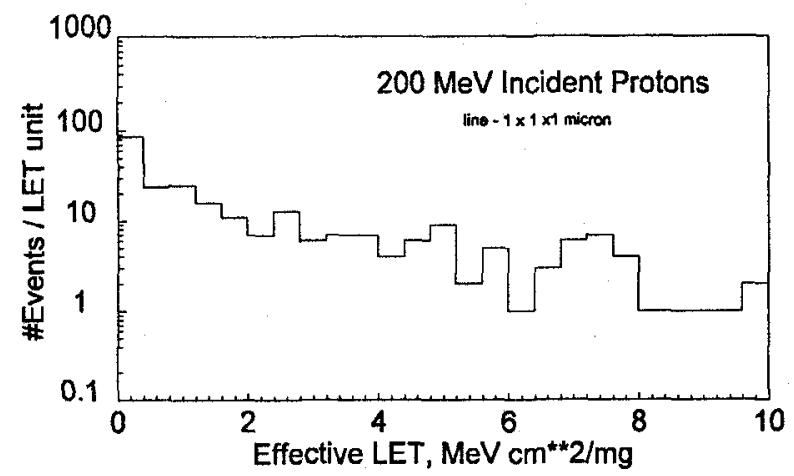

Figure 6 Differential LET spectra for thick slab embedded in bulk silicon more representative of modern technology devices $(1 \times 1 \times 1$ micron) for $200 \mathrm{MeV}$ incident protons.

IV. Risk Assessment for Heavy Ion Environments Figure 7 shows the fraction of proton events that produce a given LET. This figure was produced by integration of the differential LET spectra in Figures 5 and 6 . This shows that $10 \%$ of the events produce an LET of $8 \mathrm{MeV} \mathrm{cm} / \mathrm{mg}$ or greater. Therefore, if a component is exposed to a large fluence of protons during testing (typical proton test exposure is $10^{10}$ protons $/ \mathrm{cm}^{2}$ ) then failure modes with LET thresholds up to about $8 \mathrm{MeV} \mathrm{cm} / \mathrm{mg}$ have a good chance of being observed. In fact, some of the proton events produce LET as high as $15 \mathrm{MeV} \mathrm{cm} / \mathrm{mg}$ and failures associated with these events may be observed if the device is exposed to a large enough fluence.

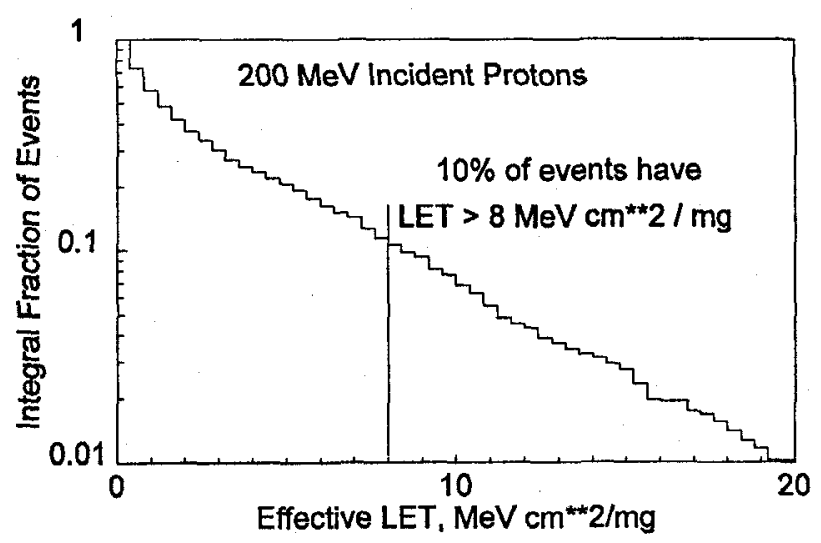

Figure 7. Fraction of events which produce at least a given LET

Therefore, if a component is tested with protons and it does not exhibit single event effects it could still be vulnerable to failures for higher LET particles which are present in the GCR's found in earth orbit. Figure 8 shows the flux of heavy ions for a Space Station orbit (51.6 degree inclination, low altitude orbit with 0.1 " of aluminum shielding). Note that only about 17 particles pass through a $1 \mathrm{~cm}^{2}$ device in a year that have LET of $8 \mathrm{MeV} \mathrm{cm} / \mathrm{mg}$ or greater. Therefore, the events that do not get detected during proton testing will not occur more than about once a month in the Space Station orbit environment.

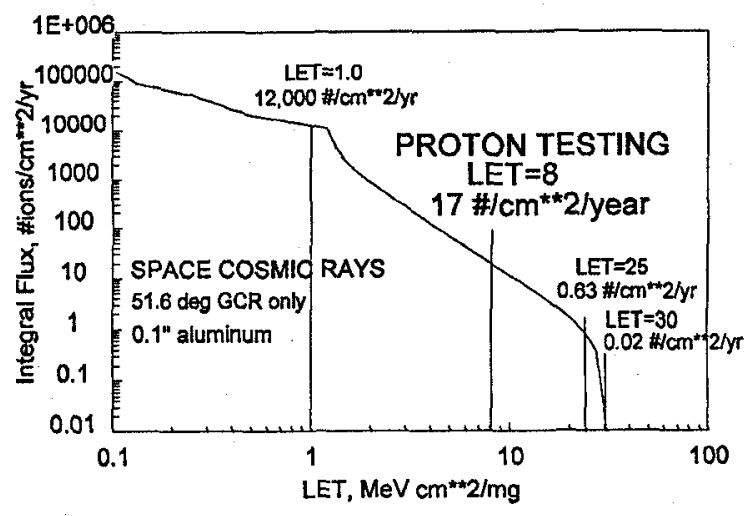

Figure 8. Integral LET Flux of heavy ions for low earth orbit showing effectiveness of proton testing 
However, for harsher heavy ion environments, the number of ions with LET $>8 \mathrm{MeV} \mathrm{cm} \mathrm{cm}^{2} / \mathrm{mg}$ is significantly larger. Table 1 shows the flux of ions with LET $>8 \mathrm{MeV} \mathrm{cm} / \mathrm{mg}$ in the natural space environment for various earth orbits (0.1" of aluminum shielding). Note that for low altitude orbits with inclination below 57 degrees, there are fewer than about one ion every few weeks for a square centimeter. For these orbits, proton screening may be all that is required for some applications.

\begin{tabular}{|l|l|l|}
\hline $\begin{array}{l}\text { Inclination } \\
\text { (degree) }\end{array}$ & $\begin{array}{l}\text { Altitude } \\
\text { (nmi) }\end{array}$ & $\begin{array}{l}\text { \# ions } / \mathrm{cm}^{2} \mathrm{yr} \\
\text { (LET>8 } \mathrm{MeV} \mathrm{cm} / \mathrm{mg})\end{array}$ \\
\hline 28.5 & 300 & $<1$ \\
\hline 51.6 & 300 & 17 \\
\hline 57 & 300 & 38 \\
\hline 90 & 300 & 140 \\
\hline 0 & 23,000 & 620 \\
\hline
\end{tabular}

Table 1 Number of ions with LET $>8 \mathrm{MeV} \mathrm{cm} / \mathrm{mg}$ that strike a one square centimeter area in a year for various earth orbits $\left(0.1^{\prime \prime}\right.$ al)

For example, an air data system that is only used one or two orbits each Shuttle mission can be tested with protons at 200 $\mathrm{MeV}$ and single event upsets can be characterized. The nominal upset rate for the South Atlantic Anomaly protons can easily be determined. If this rate satisfies the system's operation requirements, the system is probably acceptable because it's utilization is so small that the failures that could occur due to the higher LET particles $(>8 \mathrm{MeV} \mathrm{cm} / \mathrm{mg})$ will be so rare that the system redundancy can easily handle them.

In cases where components will be exposed for longer periods or for harsher heavy ion environments, it may be necessary to determine the failure modes that occur at the higher LET's by heavy ion testing, since proton testing will not trigger latchups and burnouts that have thresholds above $8 \mathrm{MeV}$ $\mathrm{cm}^{2} / \mathrm{mg}$. For example, a new integrated inertial navigation system - GPS is being procured for both Shuttle and Space Station. This is COTS equipment with no ionizing radiation pedigree. Therefore, we supplemented proton testing with system level heavy ion testing at the Alternate Gradient Synchrotron (AGS) at Brookhaven National Laboratory. The AGS provided a gold beam at $11 \mathrm{GeV} /$ nucleon and the penetration ( $10 \%$ energy loss in $15 \mathrm{~g} / \mathrm{cm}^{2}$ ) was high enough to allow system testing at a 70 degree incidence angle. This produced an effective LET in the system of $30 \mathrm{MeV} \mathrm{cm}$ / mg. For this application, this reduced the risk of an unobserved failure mode (a failure with an LET threshold of $>$ $30 \mathrm{MeV} \mathrm{cm} / \mathrm{mg}$ ) to less than one in fifty years (Figure 8 shows 0.02 ions $/ \mathrm{cm}^{2} / \mathrm{yr}$ ) for every square centimeter of sensitive device,

\section{Conclusions}

The CLUST-EVAP simulations have shown that a wide range of effective LET for slabs in silicon is obtained from the proton-silicon interaction (Figure $5 \& 6$ ). It shows that $10 \%$ of the failures observed during proton testing are produced by an effective LET of $8 \mathrm{MeV} \mathrm{cm}^{2} / \mathrm{mg}$ or greater. This shows that the failures with LET thresholds up to about $8 \mathrm{MeV} \mathrm{cm} / \mathrm{mg}$ would probably be detected in proton testing. It was shown that the error rate for failure modes that have LET thresholds $>8 \mathrm{MeV} \mathrm{cm} / \mathrm{mg}$ is limited to about 38 every year for each $\mathrm{cm}^{2}$ of sensitive cross-section for a large class of low earth orbits (mainly inclination $<57$ degrees). In many cases, this information is adequate for performance evaluation. In other cases, such as systems for Space Station that will be exposed for long periods or the harsher heavy ion environments, testing at higher LET's (at component level in vacaum or at system level at a high energy facility such as the AGS is still required.

\section{References}

[1] C. C. Foster, S.L. Casey, A.L. Johnson, P. Miesle, N. Sifri, A.H. Skees, and K. M. Murray, "Opportunities for Single Event and Other Radiation Effects Testing and Research at the Indiana University Cyclotron Facility," IEEE Nuclear Science and Radiation Effects Conference Workshop Proc., (July 1996).

[2] C. C. Foster, S.L. Casey, A.L. Johnson, P. Miesle, N. Sifri, A.H. Skees, and K. M. Murray, "Radiation Effects Test Facility at the Indiana University Cyclotron Facility, 14th International Conference on the Application of Accelerators in Research and Industry Workshop Proc. held in Denton, Texas (April 1997).

[3] W.L. Bendel and E. L. Petersen, "Proton Upsets in Orbit," IEEE Trans. Nuc. Sci. NS-30, (1983) 4481.

[4] E. L. Petersen, "The Relationship of Proton and Heavy Ion Upset Thresholds," IEEE Trans. Nuc. Sci. NS-39, (1992) 1600 .

[5] McNulty, P.J., W.G. Abdel-Kader, and G. E. Farrell, "Proton induced spallation reactions;" Radiat. Phys Chem., 43, No. 1/2 (1994) 139.

[6] Teleat, Shadia El, G.E. Farrell and P.J. McNulty, "Charge-Deposition Spectra in Thin Slabs of Silicon Induced by Energetic Protons," IEEE Trans. Nuc. Sci. NS-30, (1983), 4394.

[7] Tang, H.H.K., G.R. Srinivasan, and N. Azziz, "Cascade statistical model for nucleon-induced reactions on light nuclei in the energy range $50 \mathrm{MeV}-1 \mathrm{GeV}$,".Phys. Rev. C, 42, No. 4 (1990 October) 1598.

[8] N. Azziz, H.H.K. Tang, and G.R. Srinivasan, "A Microscopic Model of Energy Deposition in Silicon Slabs Exposed to High-Energy Protons," J. Appl. Phys. 62, No. 2 (15 July 1987), 414.

[9] Viola, Vic, Private Communication, 1995.

[10] Kwiatkowski, K., S.H. Zhou, T.E. Ward, V.E. Viola, Jr, H. Breuer, G.J. Mathews, A. Gokmen, and A.C. Mignerey, "Energy Deposition in Intermediate-Energy Nucleon-Nucleus Collisions," Phys. Rev. Letters, 50, No. 21 (23 May 1983) 1648 . 